\title{
Derangements of Sodium Homeostasis in Acute Infantile Diarrhea
}

\author{
Ismail E Haffejee* \\ Department of Paediatrics and Child Health, University of KwaZuluNatal, South Africa
}

*Corresponding author: Ismail E Haffejee, MBBCh, FCP(Paed), MD, FCFP(SA), Honorary Professor and Sessional Consultant, Department of Paediatrics and Child Health, Nelson R Mandela School of Medicine, University of KwaZuluNatal, Durban, South Africa

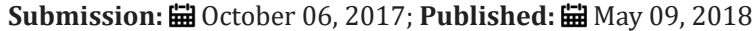

\section{Introduction}

The majority of infants with acute diarrhea have isonatraemic dehydration, where the serum sodium $(\mathrm{Na})$ concentration is within the normal range of 135 to $150 \mathrm{mEq} / \mathrm{L}$. They respond well to both oral and intravenous (IV) rehydration, the latter usually as halfstrength Darrow's solution which has a Na concentration of 65 $\mathrm{mEq} / \mathrm{L}$. Intravenous solutions with higher osmolalities such as Ringer lactate, half-normal saline or even normal saline can safely be used to treat infants showing signs of severe dehydration with or without shock. Clinical signs of dehydration will not be discussed in this paper.

Less frequently, but more ominously, one encounters either hyponatraemia ( $\mathrm{Na}$ concentration of $<130 \mathrm{mEq} / \mathrm{L}$ ) or hypernatraemia $(\mathrm{Na}>150 \mathrm{mEq} / \mathrm{L})$. Both are extremely dangerous in that they can lead to severe neurological impairment and even death. The focus of discussion in the ensuing paragraphs will be on these two electrolyte derangements.

\section{Hyponatraemia}

This occurs when there is excessive loss of sodium over water in the diarrhoeal stools, resulting in a relative hypo-osmolality of the extracellular compartment; in the brain, water will move into the cerebral cells, especially the astrocytes, due to osmotic forces, resulting in intracellular cerebral oedema, which in turn leads to a rise in intracranial pressure often manifesting with convulsions. In fact, hyponatraemia is the most common cause of generalized convulsions in a baby with acute diarrhea; this clinical scenario necessitates the immediate laboratory estimation of electrolytes to confirm the diagnosis so that appropriate treatment can be instituted urgently. It should also be remembered that increased intracranial pressure has the potential to cause herniation of the cerebellar tonsils through the foramen magnum (coning of the brain), albeit rarely.

\section{Management}

If the patient is already receiving intravenous fluids, one must ensure that the sodium concentration of the infusate is high enough.
Thus, solutions such as Ringer lactate (with a sodium concentration of $154 \mathrm{mmol} / \mathrm{L}$ ) or $5 \%$ dextrose in $0.9 \%$ saline are ideal. This means that if the baby is already on half-strength Darrow's solution this will have to be changed to one of the more concentrated solutions. Alternatively one can add $20 \mathrm{ml}$ of $8.5 \%$ sodium bicarbonate to 500 $\mathrm{ml}$ of half-Darrow's to increase its sodium concentration to 101 $\mathrm{mmol} / \mathrm{L}$. At this stage the patient will generally be out of the acute stage of severe dehydration and shock so that the rate of infusion must now be reduced since too rapid administration can result in the rare but devastating complication of osmotic demyelination syndrome, also known as central pontinemyelinolysis, which is thought to result from rapid movements of fluid in and out of the cerebral cells. The recommended rate of correction of hyponatraemia is $10 \mathrm{mmol} / \mathrm{L} / 24$ hours: this necessitates frequent electrolyte estimations, generally every 6 hours.

When water is down and salt is low,

Use strong stuff, but go very very slow!

Otherwise you may create a medical crisis

If baby develops central pontinemyelinolysis!

(With apologies to Ralph Hendrickse)

\section{Hypernatraemia}

When there is excessive loss of water over sodium in the diarrhoeal stools hypernatraemia can ensue. The classical description of this depicts an overfed, perhaps obese, baby who has been subjected to over concentrated formula feeds by an overzealous mother. However, this can also occur in breastfed infants who are well nourished, particularly in very hot tropical settings, in the absence of offering free water. In the author's experience at the King Edward VIII Hospital in Durban, South Africa, hypernatraemic dehydration has been encountered in many severely malnourished (marasmic) infants presenting with acute diarrhoea without a history of being fed over concentrated formula; the majority of such patients are HIV positive and the exact mechanism of them developing hypernatraemia is uncertain at this stage. 
The initial pathophysiological effect of hypernatraemia in the brain is shrinkage of the brain cells due to movement of water out of the cells into the extracellular space, thus increasing the intracellular osmolality. Partial compensation occurs fairly rapidly due to solute movement (mainly $\mathrm{Na}$ and chloride) into the cerebral cells; in addition, brain cells have an adaptive or protective mechanism which produces idiogenic osmoles which are osmotically active products of cellular metabolism and include taurine, glycine, glutamine, sorbitol and inositol; these have a net effect of drawing water back into the cerebral cells thus increasing intracellular osmolality and thereby limiting cell shrinkage. Remember that this adaptive mechanism takes place spontaneously without any specific treatment and often before the laboratory result of the electrolyte disturbance is known Therefore, very rapid reduction of this intracellular osmolality by infusing hypotonic solutions such as half-Darrow's will lead to an excessive amount of water moving into the cerebral cells resulting in brain swelling as a consequence of which the patient may develop seizures, signs of raised intracranial pressure and coma. This is more likely to occur if the intravenous infusion is administered too rapidly! Other serious complications of hypernatraemia include cerebral venous sinus thrombosis (a result of the increased blood viscosity) and cerebral hemorrhage caused by traction on and tearing of cerebral blood vessels bridging the brain parenchyma and the meninges as a consequence of the brain shrinkage mentioned above. Clinically, this can present as tone disturbances (e.g. paraplegia in superior sagittal sinus thrombosis), lethargy, focal neurological signs, and even convulsions.

\section{Management of hypernatremic dehydration}

If the patient was already started on oral rehydration and does not show signs of deterioration, this should be continued; in fact,good results with oral rehydration have been well documented by numerous authors and may actually be safer than IV rehydration as it is less likely to lead to a precipitous increase in intracellular water.

For the patient who is already on IV fluids one has to ensure that this is not hypo-osmolar. In other words, if half-Darrow's was initially being infused this will have to be replaced with a solution containing more sodium. This may seem paradoxical but is in fact not so considering that by this time the shrunken cerebral cells have already resumed their normal configuration and are thus liable to develop intracellular oedema if the intravenous infusion is hypo-osmolar especially if it is given too rapidly. To increase the sodium concentration of the infusate one can use one of the following strategies:

(a) Use a solution which contains a $\mathrm{Na}$ concentration of $2 / 3$ of the patient's serum Na to prevent a precipitous drop in osmolality: in the presence of metabolic acidosis one can simply add sodium bicarbonate $8 \mathrm{ml}$ of $8.5 \%$ to every $200 \mathrm{ml}$ (or $20 \mathrm{ml}$ to every $500 \mathrm{ml}$ ) of half-Darrow's which will give a $\mathrm{Na}$ concentration of $101 \mathrm{mmol} / \mathrm{L}$

(b) If there is only a mild acidosis or no acidosis, $30 \mathrm{ml}$ of $5 \%$ sodium chloride can be added to every $500 \mathrm{ml}$ of half-Darrow's

(c) Alternatively, one can replace the original half-Darrow's with either $5 \%$ dextrose in $0.9 \%$ saline or with Ringer lactate, as outlined above for hyponatraemia

Irrespective of which IV solution is being used, it must be emphasized that that the drip should run very, very slowly, not more than $10 \mathrm{ml} / \mathrm{kg} /$ hour; this infusion rate will account for deficit, maintenance requirements, as well as ongoing losses. It is preferable to plan a slow correction of the serum Na over 48 or even 72 hours, with frequent monitoring of the serum Na levels. Again, a rate of correction not exceeding about $10 \mathrm{mmol} / \mathrm{L} / 24$ hours is recommended.

Gradual rehydration, preferably oral, gradual correction of the hypernatremia, intensive frequent monitoring and looking out for any neurological complications form the cornerstone of optimal management.

Too rapid intravenous fluid administration, particularly using "hypotonic" solutions like half-Darrow's, can result in intracellular cerebral oedema. Remember that the previously shrunken brain cells quickly resume their normal configuration due to the adaptive mechanism mentioned above, which means that a slow infusion using a more concentrated solution (just as for hyponatraemia) is recommended

"Take it easy, keep your wits,

For rushing things will bring on fits!"

\section{Some Pitfalls}

1. If hyponatraemia coexists with hyperkalaemia, this may be a lab error or due to haemolysis occurring in the specimen bottle.

2. However, if repeat tests show persistent hyperkalaemia, always think of two serious conditions, namely: (a) adrenocortical insufficiency and (b) renal failure.

These entities fall beyond the scope of this article and have been mentioned here to remind readers of potential danger of missing an important diagnosis. 
Creative Commons Attribution 4.0 International License

RPN Research in Pediatrics \& Neonatology

Submit Article

\section{Research in Pediatrics \& Neonatology}

\section{Benefits of Publishing with us}

- High-level peer review and editorial services

- Freely accessible online immediately upon publication

- Authors retain the copyright to their work

- Licensing it under a Creative Commons license

- Visibility through different online platforms 